\title{
COMPLETE COVERINGS AND GALOIS CORINGS
}

\author{
TOMASZ BRZEZIŃSKI AND ADAM P. WRIGHTSON
}

\begin{abstract}
It is shown that any finite complete covering of a non-commutative algebra in the sense of Calow and Matthes (J. Geom. Phys. 32 (2000), 114-165) gives rise to a Galois coring.
\end{abstract}

\section{INTRODUCTION}

Early in the development of non-commutative geometry it was realised that an algebraic structure underlying the geometric notion of a non-commutative principal bundle is provided by a Hopf-Galois extension (cf. [12]). Motivated by the importance of principal bundles in physics, where they provide a geometric framework for gauge theories, the foundations of the theory of connections or gauge fields on Hopf-Galois extensions or quantum principal bundles were laid in [3]. Also in [3] the first non-trivial example of a connection in a quantum principal bundle, namely the q-deformed Dirac magnetic monopole potential, was described. From physics as well as (algebro) geometric point of view it is important to understand local structure of quantum principal bundles. A proposal of a definition of a locally trivial quantum principal bundle was already made in [3. These ideas were then developed more fully in the framework of $C^{*}$-algebras by Budzyński and Kondracki in [5] and then in a purely algebraic form by Calow and Matthes in [7, [8]. This has led to the formal definition of coverings and complete coverings of non-commutative algebras and also to the construction of an explicit example of a locally trivial quantum principal bundle that was later shown in [10] to be a Hopf-Galois extension.

In [1] it has been realised that a general algebraic framework for studying HopfGalois extensions and their generalisations is provided by a special class of coalgebras over non-commutative algebras or corings [14] termed Galois corings. On the other hand Kontsevich and Rosenberg [11] have proposed an approach to non-commutative algebraic geometry, whereby the structure of a non-commutative scheme is encoded in a coring of a specific kind. Such a coring is termed a cover and a non-commutative algebraic space is recovered from a quotient of a derived category of comodules of a cover. Galois corings appear as examples of covers.

The aim of this note is to make clear and explicit the connection between coverings appearing in the context of quantum principal bundles and covers of non-commutative algebraic geometry. The connection is provided by Galois corings. More specifically we show that any covering in the sense of Calow and Matthes induces a coring. This coring is a Galois coring provided the covering is complete. 


\section{Preliminaries}

We work over a field $k$. All algebras are over $k$, associative and with a unit. The unadorned tensor product is over $k$.

Let $A$ be an algebra. An $A$-bimodule $\mathfrak{C}$ is called an $A$-coring provided there exist $A$-bilinear maps $\Delta_{\mathfrak{C}}: \mathfrak{C} \rightarrow \mathfrak{C} \otimes_{A} \mathfrak{C}$ and $\varepsilon_{\mathfrak{C}}: \mathfrak{C} \rightarrow A$ such that

$$
\left(\Delta_{\mathfrak{C}} \otimes_{A} I_{\mathfrak{C}}\right) \circ \Delta_{\mathfrak{C}}=\left(I_{\mathfrak{C}} \otimes_{A} \Delta_{\mathfrak{C}}\right) \circ \Delta_{\mathfrak{C}}, \quad\left(\varepsilon_{\mathfrak{C}} \otimes_{A} I_{\mathfrak{C}}\right) \circ \Delta_{\mathfrak{C}}=\left(I_{\mathfrak{C}} \otimes_{A} \varepsilon_{\mathfrak{C}}\right) \circ \Delta_{\mathfrak{C}}=I_{\mathfrak{C}} .
$$

The map $\Delta_{\mathfrak{C}}$ is known as a coproduct and $\varepsilon_{\mathfrak{C}}$ is known as a counit of $\mathfrak{C}$. For a detailed account of the theory of corings the reader is referred to [4. An example of a coring that is most relevant to the subject of this paper can be constructed as follows [14]. First, consider an algebra map $\iota: B \rightarrow A$ (extension of algebras). Then $A$ is a $B$-bimodule via $b \cdot a \cdot b^{\prime}=\iota(b) a \iota\left(b^{\prime}\right)$, for all $a \in A, b, b^{\prime} \in B$. Consequently $A \otimes_{B} A$ is well-defined and an $A$-bimodule with the actions $a \cdot\left(a^{\prime} \otimes_{B} a^{\prime \prime}\right) \cdot a^{\prime \prime \prime}=a a^{\prime} \otimes_{B} a^{\prime \prime} a^{\prime \prime \prime}$. Furthermore, it is an $A$-coring with coproduct and counit

$$
\Delta_{A \otimes_{B} A}\left(a \otimes_{B} a^{\prime}\right)=a \otimes_{B} 1_{A} \otimes_{A} 1_{A} \otimes_{B} a^{\prime}=a \otimes_{B} 1_{A} \otimes_{B} a^{\prime}, \quad \varepsilon_{A \otimes_{B} A}\left(a \otimes_{B} a^{\prime}\right)=a a^{\prime} .
$$

$A \otimes_{B} A$ is known as the canonical or Sweedler coring associated to a ring extension $\iota: B \rightarrow A$.

An element $g$ of an $A$-coring $\mathfrak{C}$ is called a grouplike element provided $\Delta_{\mathfrak{C}}(g)=g \otimes_{A} g$ and $\varepsilon_{\mathfrak{C}}(g)=1_{A}$. Given a grouplike element $g$ one defines $g$-coinvariants of $A$ by

$$
A_{g}^{c o \mathfrak{C}}=\{b \in A \mid b \cdot g=g \cdot b\} .
$$

$A_{g}^{c o C}$ is a subalgebra of $A$, hence the obvious inclusion map gives rise to the ring extension

$A_{g}^{c o \mathfrak{C}} \rightarrow A$, and there is the corresponding Sweedler coring $A \otimes_{B} A$, with $B=A_{g}^{\text {coc }}$. A coring $\mathfrak{C}$ with a grouplike element $g$ is called a Galois coring provided the $A$-bilinear map

$$
\operatorname{can}_{A}: A \otimes_{B} A \rightarrow \mathfrak{C}, \quad a \otimes_{B} a^{\prime} \mapsto a \cdot g \cdot a^{\prime},
$$

where $B$ are $g$-coinvariants of $A$, is an isomorphism (of corings).

\section{Complete coverings and Galois corings}

The notion of a complete covering of a non-commutative algebra was introduced in [7] as a purely algebraic version of a covering of a $C^{*}$-algebra by closed ideals described in the context of quantum principal bundles in [5].

Definition 3.1. ([7) Let $B$ be an algebra. A finite family of ideals $\left(J_{i}\right)_{i \in I}$ is called a covering of $B$ if

$$
\bigcap_{i \in I} J_{i}=\{0\}
$$

Denote the factor algebras of $B$ with respect to the ideals $J_{i}, J_{i}+J_{j}$, and so on by $B_{i}, B_{i j}$, and the corresponding canonical surjections by

$$
\pi_{i}: B \rightarrow B_{i}, \quad \pi_{i j}: B \rightarrow B_{i j}, \quad \pi_{i j k}: B \rightarrow B_{i j k},
$$

etc. Obviously, there exist also surjective maps between various quotients

$$
\pi_{j_{1} \ldots j_{n}}^{i_{1} \ldots i_{m}}: B_{i_{1} \ldots i_{m}} \rightarrow B_{i_{1} \ldots i_{m} j_{1} \ldots j_{n}}, \quad b+J_{i_{1} \ldots i_{m}} \mapsto b+J_{i_{1} \ldots i_{m}}+J_{j_{1} \ldots j_{n}} .
$$


Definition 3.2. ([7] Given a covering $\left(J_{i}\right)_{i \in I}$ of $B$, define a subalgebra $B_{c}$ of $\bigoplus_{i \in I} B_{i}$,

$$
B_{c}:=\left\{\left(a_{i}\right)_{i \in I} \in \bigoplus_{i \in I} B_{i} \mid \pi_{j}^{i}\left(a_{i}\right)=\pi_{i}^{j}\left(a_{j}\right)\right\} .
$$

$B_{c}$ is known as the covering completion of $B$. A covering $\left(J_{i}\right)_{i \in I}$ is said to be complete if the map

$$
\kappa: B \rightarrow B_{c}, \quad \kappa(b)=\left(\pi_{i}(b)\right)_{i \in I}
$$

is surjective.

Note that, since the ideals forming a covering of $B$ intersect trivially, the map $\kappa$ constructed in Definition 3.2 is automatically injective. Hence, equivalently, for a covering to be complete one can require $\kappa$ be bijective.

The first main result of this note is that to any covering $\left(J_{i}\right)_{i \in I}$ of an algebra $B$, one can associate a coring that captures very closely the way in which $B$ is covered by the ideals.

Proposition 3.3. Let $\left(J_{i}\right)_{i \in I}$ be a covering of an algebra $B$ and define an algebra $A=\bigoplus_{i \in I} B_{i}$. Consider $\mathfrak{C}=\bigoplus_{i, j} B_{i j}$. $\mathfrak{C}$ is an A-bimodule with the products

$$
\left(a_{i}\right)_{i \in I} \cdot\left(a_{j k}\right)_{j, k \in I} \cdot\left(a_{l}^{\prime}\right)_{l \in I}=\left(\pi_{k}^{j}\left(a_{j}\right) a_{j k} \pi_{j}^{k}\left(a_{k}^{\prime}\right)\right)_{j, k \in I},
$$

for all $a_{i}, a_{i}^{\prime} \in B_{i}$ and $a_{j k} \in B_{j k}$. Then $\mathfrak{C}$ is an $A$-coring with the coproduct

$$
\begin{aligned}
\Delta_{\mathfrak{C}}\left(\left(\pi_{i j}\left(b_{i j}\right)\right)_{i, j \in I}\right) & =\sum_{k \in I}\left(\pi_{i l}\left(b_{i k}\right)\right)_{i, l \in I} \otimes_{A}\left(\pi_{m j}\left(\delta_{k j} 1_{B}\right)\right)_{m, j \in I} \\
& =\sum_{k \in I}\left(\pi_{n}\left(b_{n k}\right)\right)_{n \in I} \cdot\left(\pi_{i l}\left(1_{B}\right)\right)_{i, l \in I} \otimes_{A}\left(\pi_{m j}\left(\delta_{k j} 1_{B}\right)\right)_{m, j \in I} \\
& =\sum_{k \in I}\left(\pi_{i l}\left(\delta_{i k} 1_{B}\right)\right)_{i, l \in I} \otimes_{A}\left(\pi_{m j}\left(b_{k j}\right)\right)_{m, j \in I} \\
& =\sum_{k \in I}\left(\pi_{i l}\left(\delta_{i k} 1_{B}\right)\right)_{i, l \in I} \otimes_{A}\left(\pi_{m j}\left(1_{B}\right)\right)_{m, j \in I} \cdot\left(\pi_{n}\left(b_{k n}\right)\right)_{n \in I},
\end{aligned}
$$

and the counit

$$
\varepsilon_{\mathfrak{C}}\left(\left(\pi_{i j}\left(b_{i j}\right)\right)_{i, j \in I}\right)=\left(\pi_{i}\left(b_{i i}\right)\right)_{i \in I},
$$

for all $b_{i j} \in B$.

Proof. The equivalent forms of the coproduct can easily be verified. It is then clear that $\Delta_{\mathfrak{C}}$ is an $A$-bimodule map and that $\varepsilon_{\mathfrak{C}}$ is a counit. That $\Delta_{\mathfrak{C}}$ is coassociative can also be checked by a straightforward calculation (but, in the light of the discussion below this is not necessary).

There is an algebra inclusion $\iota: B \rightarrow A$ given by $b \mapsto\left(\pi_{i}(b)\right)_{i \in I}$, i.e., of the same explicit form as the map $\kappa$ in Definition [3.2. hence we can consider the corresponding canonical (Sweedler) coring $A \otimes_{B} A$. In fact the coring $\mathfrak{C}$ defined in Proposition 3.3 is isomorphic to $A \otimes_{B} A$. The isomorphism can be explicitly defined in two stages as follows.

First, for all $i, j \in I$, there is an $A$-bimodule isomorphism

$$
\Phi_{i j}: B_{i} \otimes_{B} B_{j} \rightarrow B_{i j}, \quad a \otimes a^{\prime} \mapsto \pi_{j}^{i}(a) \pi_{i}^{j}\left(a^{\prime}\right)
$$


with the inverse, for all $b \in B$,

$$
\Phi_{i j}^{-1}: \pi_{i j}(b) \mapsto \pi_{i}\left(1_{B}\right) \otimes_{B} \pi_{j}(b)=\pi_{i}(b) \otimes_{B} \pi_{j}\left(1_{B}\right) .
$$

The $\Phi_{i j}$ taken together give the $A$-bimodule isomorphism

$$
\Phi: \bigoplus_{i, j} B_{i} \otimes_{B} B_{j} \rightarrow \bigoplus_{i, j} B_{i j}, \quad \Phi=\bigoplus_{i, j} \Phi_{i j} .
$$

Second, since direct sums commute with tensor products, there is the $A$-bimodule isomorphism

$$
\Theta: \bigoplus_{i \in I} B_{i} \otimes_{B} \bigoplus_{j \in I} B_{j} \rightarrow \bigoplus_{i, j \in I} B_{i} \otimes_{B} B_{j}, \quad\left(a_{i}\right)_{i \in I} \otimes_{B}\left(a_{j}^{\prime}\right)_{j \in I} \mapsto\left(a_{i} \otimes_{B} a_{j}^{\prime}\right)_{i, j \in I}
$$

Combining $\Phi$ with $\Theta$ we obtain the $A$-bimodule isomorphism

$$
\begin{gathered}
\chi: A \otimes_{B} A \rightarrow \mathfrak{C}, \quad \chi=\Phi \circ \Theta ; \\
\left(a_{i}\right)_{i \in I} \otimes_{B}\left(a_{j}^{\prime}\right)_{j \in I} \mapsto\left(\pi_{j}^{i}\left(a_{i}\right) \pi_{i}^{j}\left(a_{j}^{\prime}\right)\right)_{i, j \in I},
\end{gathered}
$$

with the inverse, for all $b_{i j} \in B$,

$$
\chi^{-1}:\left(\pi_{i j}\left(b_{i j}\right)\right)_{i, j \in I} \mapsto \sum_{k \in I}\left(\pi_{i}\left(b_{i k}\right)\right)_{i \in I} \otimes_{B}\left(\pi_{j}\left(\delta_{j k}\right)\right)_{j \in I} .
$$

A straightforward calculation verifies that $\chi$ is an $A$-coring map (and we can deduce from this and the coassociativity of the coproduct in $A \otimes_{B} A$ that the coproduct of $\mathfrak{C}$ is coassociative).

To understand better how $\mathfrak{C}$ captures the way in which $B$ is covered by $\left(J_{i}\right)_{i \in I}$, we can use further isomorphisms

$$
\begin{gathered}
A \otimes_{B} A \otimes_{A} A \otimes_{B} A \simeq A \otimes_{B} A \otimes_{B} A \\
B_{i} \otimes_{B} B_{j} \otimes_{B} B_{k} \rightarrow B_{i j k}, \quad a_{i} \otimes_{B} a_{j} \otimes_{B} a_{k} \mapsto \pi_{j k}^{i}\left(a_{i}\right) \pi_{i k}^{j}\left(a_{j}\right) \pi_{i j}^{k}\left(a_{k}\right)
\end{gathered}
$$

and

$$
\bigoplus_{i \in I} B_{i} \otimes_{B} \bigoplus_{j \in I} B_{j} \otimes_{B} \bigoplus_{k \in I} B_{k} \simeq \bigoplus_{i, j, k \in I} B_{i} \otimes_{B} B_{j} \otimes_{B} B_{k}
$$

to identify $\mathfrak{C} \otimes_{A} \mathfrak{C}$ with the $A$-bimodule of "triple intersections" $\mathfrak{D}=\bigoplus_{i, j, k \in I} B_{i j k}$. In terms of this identification, the coproduct takes the following simple form, for all $a_{i j} \in$ $B_{i j}$

$$
\Delta_{\mathfrak{C}}\left(\left(a_{i j}\right)_{i, j \in I}\right)=\left(\pi_{j}^{i k}\left(a_{i k}\right)\right)_{i, j, k \in I}
$$

As an example of $\mathfrak{C}$ consider first a (topological) space $X$ covered by a finite family of (open) sets $\left(U_{i}\right)_{i \in I}$. Let $B$ be an algebra of functions on $X, B=\mathcal{O}(X)$. The algebra $B$ is then covered by the ideals $J_{i}=\left\{f \in \mathcal{O}(X) \mid \forall x \in X \backslash U_{i}, f(x)=0\right\}$, so that $B_{i}=\mathcal{O}\left(U_{i}\right)$, and each of the $\pi_{i}$ is simply the restriction of a function on $X$ to a function on $U_{i}, \pi_{i}(f)=\left.f\right|_{U_{i}}$. In addition, $B_{i j}=\mathcal{O}\left(U_{i} \cap U_{j}\right)$. The algebra $A=\bigoplus_{i \in I} B_{i}$ can be identified with the algebra of functions on the disjoint union $\bigsqcup_{i \in I} U_{i}, A=\mathcal{O}\left(\bigsqcup_{i \in I} U_{i}\right)$. Furthermore, $\mathfrak{C}=\bigoplus_{i, j \in I} B_{i j}$ can be identified with the algebra of functions on the disjoint union of intersections $\bigsqcup_{i, j \in I} U_{i} \cap U_{j}$, i.e., $\mathfrak{C}=\mathcal{O}\left(\bigsqcup_{i, j \in I} U_{i} \cap U_{j}\right)$, and also $\mathfrak{D}=\mathcal{O}\left(\bigsqcup_{i, j, k \in I} U_{i} \cap U_{j} \cap U_{k}\right)$. The coproduct and the counit come out as, for all $f \in \mathcal{O}\left(\bigsqcup_{i, j \in I} U_{i} \cap U_{j}\right)$,

$$
\left.\Delta_{\mathfrak{C}}(f)\right|_{U_{i} \cap U_{j} \cap U_{k}}=\left.f_{i k}\right|_{U_{i} \cap U_{j} \cap U_{k}},\left.\quad \varepsilon_{\mathfrak{C}}(f)\right|_{U_{i}}=\left.f\right|_{U_{i} \cap U_{i}}
$$


where $f_{i k}=\left.f\right|_{U_{i} \cap U_{k}}$. In this way we obtain an example of coring described in [1].

The following theorem establishes a relationship between the notions of complete coverings and Galois corings and also gives the criterion, when a covering is complete.

Theorem 3.4. Let $\left(J_{i}\right)_{i \in I}$ be a covering of an algebra $B, B_{i}=B / J_{i}$, and let $A=$ $\bigoplus_{i \in I} B_{i}$. Let $\mathfrak{C}=\bigoplus_{i, j} B_{i j}$ be the associated $A$-coring constructed in Proposition 3.3 . Set $g=\left(\pi_{i j}\left(1_{B}\right)\right)_{i, j \in I}$.

(1) If $\left(J_{i}\right)_{i \in I}$ is a complete covering of $B$, then $(\mathfrak{C}, g)$ is a Galois coring.

(2) If $A$ is a faithfully flat left or right $B$ module, then $\left(J_{i}\right)_{i \in I}$ is a complete covering of $B$.

Proof. First note that $g$ is a grouplike element and that it is the image of the grouplike element $1_{A} \otimes_{B} 1_{A}$ of the Sweedler $A$-coring $A \otimes_{B} A$ under the isomorphism $\chi$. Since $\pi_{i j}\left(1_{B}\right)$ is the unit in $B_{i j}$, the forms of the right and left multiplications in $\mathfrak{C}$ immediately imply that, for all $\left(a_{i}\right)_{i \in I} \in A$,

$$
\left(a_{i}\right)_{i \in I} \cdot g=\left(a_{i}\right)_{i \in I} \cdot\left(\pi_{j k}\left(1_{B}\right)\right)_{j, k \in I}=\left(\pi_{k}^{j}\left(a_{j}\right)\right)_{j, k \in I},
$$

and

$$
g \cdot\left(a_{i}\right)_{i \in I}=\left(\pi_{j k}\left(1_{B}\right)\right)_{j, k \in I} \cdot\left(a_{i}\right)_{i \in I}=\left(\pi_{j}^{k}\left(a_{k}\right)\right)_{j, k \in I} \cdot
$$

Therefore $\left(a_{i}\right)_{i \in I} \in A_{g}^{c o \mathfrak{C}}$ if and only if $\left(a_{i}\right)_{i \in I} \in B_{c}$, where $B_{c}$ is a covering completion of $B$. Thus

$$
B_{c}=A_{g}^{c o C}
$$

In view of the isomorphism $\chi$ this identification of $B_{c}$ as $g$-coinvariants immediately implies assertion (1).

The assertion (2) follows by [6, Theorem 4.12] (cf. 99, Theorem 3.10]). We present a direct proof based on the proof of [15, Lemma 4.2]. Recall that $A_{g}^{c o c}=B_{c}$ and that $B$ is a subalgebra of $B_{c}$ via $\kappa: B \rightarrow B_{c}$. Note that

$$
\chi\left(A \otimes_{B} B_{c}\right)=A \cdot g, \quad \chi\left(B_{c} \otimes_{B} A\right)=g \cdot A .
$$

Note further that $g \cdot A \simeq A$ as a $(B, A)$-bimodule and $A \cdot g \simeq A$ as an $(A, B)$-bimodule via the counit $\varepsilon_{\mathfrak{C}}$. Since $\chi$ is an isomorphism, the map

$$
A \otimes_{B} B_{c} \stackrel{\chi}{\longrightarrow} A \cdot g \stackrel{\varepsilon_{\mathfrak{C}}}{\longrightarrow} A, \quad a \otimes_{B} b \mapsto a b
$$

is an isomorphism of $(A, B)$-bimodules, and the map

$$
B_{c} \otimes_{B} A \stackrel{\chi}{\longrightarrow} g \cdot A \stackrel{\varepsilon_{\mathfrak{C}}}{\longrightarrow} A, \quad b \otimes_{B} a \mapsto b a
$$

is an isomorphism of $(B, A)$-bimodules. These lead to the factorisations of the identity maps

$$
A \stackrel{\simeq}{\longrightarrow} B \otimes_{B} A \stackrel{\kappa \otimes_{B} A}{\longrightarrow} B_{c} \otimes_{B} A \stackrel{\simeq}{\longrightarrow} A, \quad A \stackrel{\simeq}{\simeq} A \otimes_{B} B \stackrel{A \otimes_{B} \kappa}{\longrightarrow} A \otimes_{B} B_{c} \stackrel{\simeq}{\longrightarrow} A,
$$

implying that $\kappa \otimes_{B} A$ and $A \otimes_{B} \kappa$ are isomorphisms. Thus if $A$ is faithfully flat as either left or right $B$-module, then $\kappa: B \rightarrow B_{c}$ is an isomorphism, hence $\left(J_{i}\right)_{i \in I}$ is a complete covering of $B$, as claimed.

Corollary 3.5. Let $\left(J_{i}\right)_{i \in I}$ be a complete covering of an algebra $B, B_{i}=B / J_{i}$, and let $A=\bigoplus_{i \in I} B_{i}$. If $A$ is a projective left (resp. right) $B$-module, then $A$ is a faithfully flat left (resp. right) B-module. 
Proof. By Theorem $3.4(1), \mathfrak{C}=\bigoplus_{i, j \in I} B_{i j}$ is a Galois $A$-coring, hence $A$ is a principal right (resp. left) $\mathfrak{C}$-comodule in the sense of [2, Definition 4.1]. By [2, Theorem 4.3] (resp. the left-handed version of [2, Theorem 4.3]), $A$ is a faithfully flat left (resp. right) $B$-module.

In particular, Theorem 3.4 implies that if $\left(J_{i}\right)_{i \in I}$ is a covering of an algebra $B$ such that $A$ is a faithfully flat left $B$-module (e.g., if $\left(J_{i}\right)_{i \in I}$ is a complete covering and ${ }_{B} A$ is projective by Corollary 3.5$)$, then $\mathfrak{C}$ is a flat left $A$-module and the induction functor $-\otimes_{B} A$ is an equivalence from the category of right $B$-modules to the category of right $\mathfrak{C}$ comodules by [1, Theorem 5.6]. Following Kontsevich and Rosenberg, $\mathfrak{C}$-comodules are understood as quasi-coherent sheaves on a non-commutative space. Since the category of comodules (quasi-coherent sheaves) is equivalent to a module category, the underlying space is a non-commutative affine space with the coordinate ring $B$ (cf. [13, p. 2134]).

\section{REFERENCES}

[1] T. Brzeziński. The structure of corings. Induction functors, Maschke-type theorem, and Frobenius and Galois-type properties, Alg. Rep. Theory, 5 (2002), 389-410.

[2] T. Brzeziński, Galois comodules, Preprint arXiv:math.RA/0312159 v. 3 (2004), J. Algebra to appear.

[3] T. Brzeziński and S. Majid, Quantum group gauge theory on quantum spaces, Commun. Math. Phys. 157 (1993), 591-638. Erratum 167 (1995), 235.

[4] T. Brzeziński and R. Wisbauer Corings and Comodules. Cambridge University Press, Cambridge, 2003.

[5] R.J. Budzyński and W. Kondracki, Quantum principal bundles: topological aspects, Rep. Math. Phys. 37 (1996), 365-385.

[6] S. Caenepeel and E. De Groot and J. Vercruysse, Galois theory for comatrix corings: Descent theory, Morita theory, Frobenius and separability properties, Preprint arXiv:math.RA/0406436 (2004), Trans. Amer. Math. Soc. to appear.

[7] D. Calow and R. Matthes, Covering and gluing of algebras and differential algebras, J. Geom. Phys., 32 (2000), 114-165.

[8] D. Calow and R. Matthes, Connections on locally trivial quantum principal fibre bundles, J. Geom. Phys., 41 (2002), 114-165.

[9] L. El Kaoutit and J. Gómez-Torrecillas. Comatrix corings: Galois corings, descent theory, and a structure theorem for cosemisimple corings. Math. Z. (2003), 244:887906.

[10] P.M. Hajac, R. Matthes and W. Szymański. A locally trivial quantum Hopf fibration, Algebras and Rep. Theory 6 (2003), 169-192.

[11] M. Kontsevich and A.L. Rosenberg, Non-commutative smooth spaces, [In:] The Gelfand Mathematical Seminars, 1996-1999, Gelfand Math. Sem., Birkhäuser, Boston, MA, 2000, pp. 85-108.

[12] H.-J. Schneider, Principal homogeneous spaces for arbitrary Hopf algebras, Israel J. Math 72 (1990), 167-195.

[13] S.P. Smith, Subspaces of non-commutative spaces, Trans. Amer. Math. Soc. 354 (2002), $2131-2171$.

[14] M. Sweedler, The predual theorem to the Jacobson-Bourbaki theorem, Trans. Amer. Math. Soc. 213 (1975), 391-406.

[15] M. Takeuchi, On extensions of formal groups by $\mu^{A}$, Comm. Algebra 5 (1977), 14391481.

Department of Mathematics, University of Wales Swansea, Singleton Park, SWANSEA SA2 8PP, U.K.

E-mail address: T.Brzezinski@swansea.ac.uk (T Brzezinski)

E-mail address: maapw@swansea.ac.uk (AP Wrightson) 